\title{
Conséquences sanitaires de l'accident de Three Mile Island
}

\author{
M. BERTIN (*)
}

L'accident de la centrale nucléaire de Harrisburg s'est produit le 28 mars 1979, il y a donc environ un an. Il a fait l'effet d'une bombe dans les mass media, mais quelles en ont été les conséquences sanitaires?

Les différents rapports publiés à ce propos [1-3] montrent qu'elles ont été pratiquement nulles, car les trois enceintes de protection ont joué parfaitement leur rôle malgré les erreurs commises et les défaillances du matériel et des systèmes de contrôle.

L'irradiation du personnel, du 28 mars au 7 avril (c'est-à-dire au cours des manœuvres effectućes pendant l'accident et les premiers jours qui ont suivi), a été la suivante :

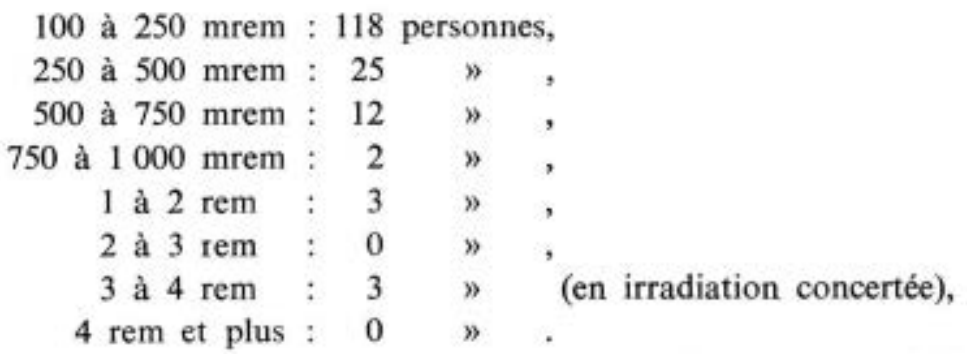

Le rapport KEMENY [3] indique pour la période du 28 mars au 30 juin des doses plus importantes.

Environ 1000 travailleurs ont reçu des doses mensuelles supérieures à $50 \mathrm{mrem}$. La dose collective pour ces mêmes travailleurs serait de 1000 homme-rem soit, en moyenne, 1 rem pour cette période de 3 mois. La limite trimestrielle n'a été dépassée que pour 3 d'entre eux (voir ci-dessus). De plus, 2 agents ont reçu, l'un 50 rem, l'autre 150 rem sur les mains.

Les doses reçues par les agents de la centrale sont donc restées à peu près dans les limites des doses admissibles pour les travailleurs.

(*) E.D.F., Comité de Radioprotection, 71, rue de Miromesnil, 75384 Paris Cedex 08.

RADIOPRoteCtION, voL. 15 - 0033-8451/1980/89/\$ 5.00/C Bordas-Dunod. 
Les vrais problèmes se poseront ultérieurement lorsque le sort de la centrale aura été fixé et qu'il faudra appliquer les mesures décidées, quelles qu'elles soient.

Notre connaissance des doses reçues par la population est, bien entendu, beaucoup moins précise.

De très nombreuses mesures ont été effectuées. La cartographie des courbes isodoses a été établie et, compte tenu de la répartition de la population, la dose intégrale reçue, exprimée en homme-rem, a pu être calculée. La période retenue est celle du 28 mars au 7 avril; au-delà, le niveau d'irradiation est trop faible pour être valablement pris en considération.

Les doses de rayonnement $\gamma$ reçues par chaque habitant sur l'ensemble du corps ont été intégrées pour l'ensemble des 2 millions d'habitants vivant dans un cercle de $80 \mathrm{~km}$ de rayon autour de la centrale. Cinq estimations ont donné respectivement $1600,2000,2800,3300$ et 5300 homme-rem; la valeur retenue en général est celle de 3300 homme-rem, soit une irradiation individuelle moyenne de 1,6 mrem (l'irradiation ultérieure au 7 avril n'augmenterait pas cette valeur de plus de $0,03 \mathrm{mrem}$ ).

Ceci, bien entendu, ne donne aucune idée de la répartition des doses, mais si ses membres étaient restés sur place $(5,6 \mathrm{~km}$ au nord de la centrale), le groupe le plus irradié aurait reçu environ $80 \mathrm{mrem}$.

En tenant compte des méthodes de calcul habituellement admises (C.I.P.R., U.N.S.C.E.A.R., B.E.I.R., etc.) on peut estimer que, pour cette population de deux millions de sujets, les conséquences seraient au maximum d'un cancer mortel (en réalité 0,3 ou 0,7 suivant les estimations) et une anomalie génétique grave (en réalité 0,3 ou 0,7 ). Ce cancer et cette anomalie génétique sont à comparer aux 400000 décès par cancer et aux 120000 anomalies génétiques qui surviendront naturellement dans cette même population.

A l'irradiation $\gamma$ s'ajoutent les effets :

- de l'irradiation $\beta$ du xénon 133 (d'après le rapport de l'Académie des Sciences [1], la probabilité d'un seul cancer cutané mortel dans cette population serait de 0,01 ; elle est donc infime),

- de la dose additionnelle aux poumons par inhalation de l'air radioactif inhalé (rayonnement $\gamma$ et $\beta$ ) qui irradie de l'intérieur (le risque ajouté serait de $1 \mathrm{p}$. cent du risque de cancérisation dû à l'irradiation externe qui est, elle-même inférieure à 1 cas pour l'ensemble de la population et pour la vie entière), l'importance est donc la même que dans le cas précédent,

- de la contamination par les iodes : la concentration maximale dans l'air, ceci pendant 2 ou 3 jours, a augmenté jusqu'à $1 / 10$ de la C.M.A. population puis elle a rapidement diminué; sur 264 analyses du lait, faites du 28 mars au 4 avril, 196 furent négatives et 68 positives, la contamination moyenne des résultats positifs représentait moins de 1/50 de la C.M.A. en France.

Les conséquences biologiques de l'accident de Three Mile Island ont donc été infimes, voire nulles. Cependant, la dose collective, que recevront les travailleurs qui auront à appliquer les mesures décidées pour l'avenir de la centrale, posera peut-être des problèmes plus sérieux. 


\section{BIBLIOGRAPHIE}

[1] Auger P., Bernard J., Gautheret R., Kastler A., Latarjet R. et Nefl L. Rapport du Comité de l'Académic des Sciences institué à la suite de l'accident survenu à la centrale nucléaire de Three Mile Island. Paris, Académie des Sciences, 1979.

[2] Êtats-Unis. Nuclear Regulatory Commission, Environmental Protection Agency, Department of Health, Education and Welfare. Preliminary dose and health impact of the accident at the Three Mile Island nuclear station by the had hoc population dose assessment group. Washington, Nuclear Regulatory Commission, 1979.

[3] Êtats-Unis. The President's Commission on the accident at Three Mile Island (dit rapport KemenY), Washington, octobre 1979. 